\title{
DIFFERENCES IN THE DYNAMICS OF CHANGES IN THE CONCENTRATION OF KYNURENIC ACID IN THE BLOOD SERUM OF DEPRESSED PATIENTS TREATED WITH ELECTROCONVULSIVE THERAPY
}

\author{
Marcin Olajossy $^{1}$, Bartosz Olajossy ${ }^{2}$, Emilia Potembska $^{3}$, Nikodem Skoczeń ${ }^{1}$, \\ Sebastian Wnuk ${ }^{2}$ \& Ewa Urbańska ${ }^{2}$ \\ ${ }^{1} 2^{\text {nd }}$ Department of Psychiatry and Psychiatric Rehabilitation Medical University of Lublin, Lublin, Poland \\ ${ }^{2}$ Chair and Department of Experimental and Clinical Pharmacology, \\ Laboratory of Cellular and Molecular Pharmacology, Lublin, Poland \\ ${ }^{3}$ Department of Psychiatric Nursing Medical University of Lublin, Lublin, Poland
}

received: 12.10.2016;

revised: 3.1.2018;

accepted: 23.8 .2018

\section{SUMMARY}

Background: Nowadays, depression is a serious clinical problem, as the prevalence of its various forms increases and there are growing numbers of patients with severe forms of depression and treatment-refractory depression. Depressed patients have been observed to have decreased levels of kynurenic acid (KYNA), which is the only endogenous antagonist of ionotropic N-methyl-Daspartate (NMDA) receptors. The aim of this study was to identify subgroups of patients differing in the dynamics of changes in KYNA concentration during electroconvulsive therapy (ECT).

Subjects and methods: The study included 32 patients with an ICD-10 diagnosis of a major depressive episode receiving ECT treatment and 48 healthy volunteers. Blood serum KYNA concentrations were determined using HPLC-based methods, and clinical assessment was performed using the Montgomery-Asberg Depression Rating Scale (MADRS) and the Clinical Global Impressions (CGI) Scale.

Results: Three subgroups of patients with depression were identified which differed in baseline KYNA levels and in the dynamics of changes in blood serum KYNA concentrations during and after ECT.

Conclusions: The largest number of patients with clinical improvement (83\%) was observed in the subgroup with significantly lower pre-treatment, treatment and post-treatment KYNA concentrations than those determined in the control group. This subgroup of patients also showed the lowest dynamics of changes in KYNA concentrations during ECT. Clinical improvement was observed in $75 \%$ of depressed patients who had insignificantly lower pre-treatment levels of KYNA and slightly higher levels of this acid after 6 and 12 ECT sessions than controls. The smallest number of patients with clinical improvement (50\%) was observed in the subgroup of patients who had similar pre-treatment and treatment concentrations of KYNA to controls and whose post-treatment concentrations of KYNA were significantly lower than those of healthy individuals.

Key words: kynurenic acid - depression - electroconvulsive therapy

$* * * * *$

\section{INTRODUCTION}

Studies conducted so far have shown that increased activation of N-methyl-D-aspartate (NMDA) receptors, which induces excitotoxicity, is one of the factors contributing to the development of depression (Diazgranados et al. 2010, Zarate et al. 2006). Raol et al. (2001) have observed that excessive activation of excitatory amino acid receptors leads to neurodegenerative changes. The role of NMDA receptors in the mechanism of action of antidepressants and electroconvulsive therapy (ECT) has been confirmed in animal (Papp \& Moryl 1994, Skolnick et al. 1996, Bobula et al. 2003) and human studies (Pittaluga et al. 2007, Michael et al. 2003). It has been shown that selective serotonin reuptake inhibitors (SSRI) (Skolnick et al. 1996, Pittaluga et al. 2007) and selective noradrenaline reuptake inhibitors (Pittaluga et al. 2007) reduce the function of NMDA receptors. According to Michael et al. (2003), ECT sessions in patients with depression also reduce the effect of these receptors. The results obtained have allowed researchers to broaden the monoaminergic conception of the etiology and treatment of depression to include the effects associated with changes in the glutamatergic system. These data indicate that inhibition of the activity of NMDA receptors can be used as one of the methods for treating diseases caused by excessive activation of the glutamatergic system.

Recent clinical trials have shown that a single dose of ketamine administered intravenously causes a rapid reduction in the severity of depressive symptoms in patients with treatment-resistant depression, both endogenous and in bipolar disorder (Zarate et al. 2006, Rybakowski et al. 2013, Price et al. 2009). It has been found that blockade of NMDA receptors by ketamine inactivates eukaryotic elongation factor 2 kinase $(\mathrm{eEF} 2 \mathrm{~K})$, resulting in reduced phosphorylation of eukaryotic elongation factor 2 (eEF2) and unlocking of 
the translation of the brain-derived neurotrophic factor (BDNF), leading to increased levels of BDNF (Kavalali \& Monteggia 2012). However, the use of ketamine is limited due to its psychomimetic effects (Kavalali \& Monteggia 2012). It has been observed that the expression of BDNF in the hippocampus increases under the influence of antidepressants (Chen et al. 2001). Ketamine blocks NMDA receptors through an interaction with sites thought to be located within the ion channel pore region (Orser et al. 1997). It was shown recently that inclusion of the NR3B subunit does not alter the ketamine sensitivity of recombinant NR1/NR2 receptors expressed in oocytes (Yamakura et al. 2005). Erhardt et al. (2003) described that in anaesthetized rats, pharmacologically elevated KYNA concentration (by means of PNU 156561A) was associated with increased firing rate and burst firing activity of midbrain DA neurons. Similar alterations in basal firing characteristics are also observed following systemic administration of PCP or ketamine, indicating per se that elevated levels of brain KYNA is associated with psychotomimetic effects.

A vast majority of brain neurons and synapses are the part of glutamatergic system, and glutamate synaptic transmission largely mediates both cognition and emotion, two brain functions that seem to be inextricably linked (Pessoa 2008). Moreover, compelling evidence from clinical studies suggest that glutamate transmission is abnormally regulated in a number of limbic, cortical areas in the brains of depressed individuals. Furthermore, increasing evidence from a lot of studies suggest the aberrant glutamatergic signaling is associated with maladaptive changes in the structure and function of excitatory circuitry, e.g., the cytoarchitectural and volumetric changes measured by postmortem histopathology and magnetic resonance imaging (MRI) (Campbell \& MacQueen 2006, Konarski et al. 2008, Koolschijn 2009, Lorenzetti et al. 2009). Glutamate and its receptors are involved in the pathophysiology of mood disorders and have recently emerged as potential targets for the pharmacotherapy of depression. In rats, Fumagalli et al. (2010), observed plasticity changes of the glutamatergic system evoked by electroconvulsive shock (ECS), which represents the most effective therapy for patients who are refractory to antidepressants. Chronic ECS produced a marked increase in the phosphorylation of the regulatory NMDA receptor subunit NR2B (Ser1303) and the AMPA receptor subunit GluR-A (Ser831) in the hippocampus, with no effects on the obligatory subunit NR1. No effects were found on total receptor subunit expression levels. Authors suggest that, at least in part, ECS exerts its clinical activity through the modulation of the glutamatergic synapses, via potentiation of AMPA currents mediated by GluR-A (Ser831) phosphorylation, and a reduction of NMDA receptor activity through the phosphorylation of NR2B (Ser1303), presumably uncoupling NR2B from its signalling partner CaMKII.
Kynurenic acid (KYNA) has the ability to competitively inhibit the strychnine-insensitive glycine site in the NMDA receptor complex and non-competitively inhibit acetylcholine receptor $\alpha-7$ (Hilmas et al. 2001). Raol et al. (2001) have shown that KYNA has neuroprotective and anti-convulsive properties.

Over $90 \%$ of tryptophan is metabolized in the human body along the kynurenine pathway (Thackray et al. 2008). Tryptophan is converted under the influence of tryptophan 2,3-dioxygenase (TDO) and indoleamine 2,3-dioxygenase (IDO) to L-kynurenine (KYN) (Myint 2012). KYN can be transformed into KYNA by kynurenine aminotransferase (KATS), into anthranilic acid (AA) by kynureninase and into 3-hydroxykynurenine (OH-KYN) by kynurenine 3-hydroxylase. Anthranilic acid and 3-hydroxykynurenine can be converted to quinolinic acid (QUIN) (Myint \& Kim 2003). According to Myint and Kim (2003) (16), 3-hydroxykynurenine (OH$\mathrm{KYN}$ ) and quinolinic acid are neurotoxic. These researchers (Myint \& Kim 2003) have found that depression is associated with disturbances in the kynurenine pathway and that it increases the synthesis of neurotoxic metabolites at the expense of the neuroprotective KYNA pathway. So far, studies seem to confirm the neurodegenerative hypothesis, as depressive patients have been reported to have significantly lower concentrations of KYNA than healthy persons (Myint et al. 2007, Olajossy 2010, Maes et al. 2011, Olajossy et al. 2014).

ECT, according to Michael et al. (2003), inhibits NMDA receptors. There are few studies on the possible impact of ECT on the level of KYNA (Olajossy 2010). Earlier results obtained by Olajossy (2010) indicate that division of patients into subgroups characterized by different dynamics of changes in the concentrations of KYNA during ECT may be used as a clue to therapy and allow accurate prediction of changes in the patients' clinical condition at follow-up. Pfleiderer et al. (2003) assessed the effect of successful electroconvulsive therapy (ECT) on glutamate/glutamine levels in the anterior cingulum. Authors observed significantly reduced glutamate/glutamine levels in the patients' left cingulum compared to healthy controls. In ECT responders, in contrast to non-responders, glutamate/glutamine levels normalized and then did not differ statistically from controls. Severe depression seems to be associated with a glutamate/glutamine deficit and increasing glutamate/ glutamine may be an important mechanism of ECT action. After ECT, the glutamate contents decreased, and NMDA-NR2B expression up-regulated, the depression symptoms improved, and the spatial memory worsened simultaneously. Propofol inhibited the excessive decrease of glutamate and excessive up-regulation of NMDA-NR2B caused by ECT, and both the depression symptoms and the spatial memory of depressed rats improved (Dong et al. 2010).

The aim of the study was to isolate subgroups of patients with different dynamics of changes in blood serum KYNA concentrations during ECT. 


\section{SUBJECTS AND METHODS}

\section{Subjects}

The study group comprised 32 patients (19 women and 13 men) with a mean age of $49.41(\mathrm{SD}=12.73)$ years, with a diagnosis of a major depressive episode without psychotic symptoms or with psychotic symptoms according to ICD-10, in the course of recurrent depressive disorder (F33). 28 patients were included into the analysis. Total number of patient was 32 , but 4 samples were not suitable because of technical reasons. The subjects were in-patients of the Department of Psychiatry. The mean duration of symptoms was 11.69 $(\mathrm{SD}=8.95)$ years, the mean number of phases of the disease was $4.53(\mathrm{SD}=2.91)$ and the mean duration of the current phase of depression was 4.95 ( $\mathrm{SD}=3.23$ ) months. The control group consisted of 48 age-matched healthy subjects. The study was approved by the Clinical Research Ethics Committee of the Medical University of Lublin, Poland (decision KE-0254/3/2006).

\section{Methods}

\section{The ECT procedure}

Patients were referred for ECT in accordance with relevant procedures after they had given their informed consent, had undergone an ECG, an EEG and laboratory tests to asses their physical state, and had been consulted by a neurologist and an internist. In accordance with the accepted principles, all patients referred for ECT were discontinued from all drugs that significantly affected the seizure threshold - this excluded the use of barbiturates, benzodiazepines, lithium and other moodstabilizing drugs. The majority of the patients received antidepressants; most often, they were treated with a monotherapy of SSRIs, as this method is considered to be both safe and effective (Zyss 2009). This procedure was mostly a continuation of existing treatment. Recruitment of a "pure" group of patients who receive ECT alone is difficult and may raise ethical objections.

The therapeutic procedures for the application of ECT did not deviate from the standards recommended in Poland (Zyss 2009). Bilateral ECT was performed using a Spectrum 500Q device in the fronto-temporal position, at suggested stimulus doses. Anesthetic premedication was used, namely, general anesthesia and muscle relaxation (injections of thiopental, succinylcholine and atropine). During premedication and after ECT, the patients were oxygenated. The treatment included 12 ECT sessions, two sessions per week.

\section{Laboratory tests}

\section{Material for laboratory tests}

All patients enrolled in the study gave their informed consent to venous blood sampling. Blood was collected four times: before ECT and after the first, sixth and twelfth ECT sessions.
$5.0 \mathrm{~mL}$ samples of native blood were drawn from the median cubital vein before the first ECT and half an hour after the first, sixth and twelfth (final) ECT. After collecting, the blood was centrifuged for 15 minutes at $3500 \mathrm{rpm}$, and then the supernatant was collected and frozen at $-72^{\circ} \mathrm{C}$.

The content of KYNA in serum was assessed at the Department of Experimental and Clinical Pharmacology of the Medical University of Lublin, using a Varian Pro Star 210 liquid chromatograph (California, USA). The chemical reagents used during the chromatographic evaluation of KYNA content in the test samples were from Baker BV (Deventer, the Netherlands). The supernatant was frozen using an Innova U-101 ultra low temperature lab freezer.

The material for analysis was prepared using a modified method by Turski et al. (1988). This method is based on the assumption that at appropriate $\mathrm{pH}$ values, KYNA is retained and then washed off into an ion exchange layer. Owing to this process, KYNA concentrations increase to values that can be detected during further analysis. Equivalent volumes of serum were mixed with $8 \%$ perchloric acid, stirred and centrifuged for $20 \mathrm{~min}$ at $4^{\circ} \mathrm{C}$ at $12000 \mathrm{rpm}$. The following chemical reagents were used: Dowex 50-W, double-distilled water and $0.1 \mathrm{~N}$ hydrochloric acid. Pasteur columns, $0.5 \mathrm{~cm}$ in diameter, were filled with $0.3 \mathrm{ml}$ of a $50 \%$ Dowex suspension in double-distilled water $(\mathrm{v} / \mathrm{v})$, and then with $1 \mathrm{ml}$ of $0.1 \mathrm{~N}$ hydrochloric acid. $1 \mathrm{ml}$ of undiluted samples was added to the Pasteur columns thus prepared. Then the columns were washed once again with $1 \mathrm{ml}$ of $0.1 \mathrm{~N}$ hydrochloric acid and $1 \mathrm{ml}$ of double-distilled water. Samples were deproteinized using Millipore syringe filters.

\section{Quantification of KYNA}

Eluted KYNA was subjected to high-performance liquid chromatography (HPLC) and quantified fluorimetrically (Varian HPLC system; ESA catecholamine HR$80.3 \mathrm{~mm}, \mathrm{C} 18$ reverse-phase column), as previously described (Urbańska et al. 1007). The mobile phase ( $\mathrm{pH}$ 6.2) contained $250 \mathrm{mM}$ zinc acetate, $50 \mathrm{mM}$ sodium acetate and $4 \%$ acetonitrile. The mean retention time of KYNA was $3.5 \mathrm{~min}$ with $1 \mathrm{ml} / \mathrm{min}$ mobile phase flow. Each chromatographic assay was preceded by measurements of standardized concentrations of KYNA $(0.2,0.4,0.6,0.8$ and $1.0 \mathrm{pmol}$ ) in order to obtain a calibration curve. The concentration of KYNA was expressed as pmol.

\section{Clinical rating scales}

The severity of depressive disorders was assessed on the Montgomery-Åsberg Depression Rating Scale (MADRS) (Montgomery \& Åsberg 1979). It is a sixpoint scale for assessing depression, in particular the socalled endogenous type. MADRS shows good agreement with other psychometric instruments for depression rating and is often used in clinical trials which test the effecti- 
veness of treatment. Severity of depression according to the MADRS score is as follows: 0-11 no depressive symptoms, 12-19 mild depression, 20-28 moderate depression, 29-43 severe depression, 44-60 very severe depression. The Clinical Global Impression (CGI) scale (Guy 1976) was also used in this study. CGI provides a rough assessment of the severity of a mental disorder.

Improvement of mental state was defined as a $30 \%$ reduction in symptoms on MADRS with simultaneous reduction, by two or more points, of symptom severity measured on the CGI scale after 12 ECT sessions.

\section{Statistical tests}

The results were analyzed statistically using STATISTICA 10.0 PL software. Because the measurements were characterized by high skewness, results were presented as median, a measure of central tendency. Equality of distribution for each variable within normal distribution groups was tested using the Lilliefors version of the Kolmogorov-Smirnov test as well as the ShapiroWilk test. Because the test variables did not have a normal distribution, non-parametric tests were used for further analysis. These tests are resistant to deviations from the assumptions of normality of distribution and heterogeneity of variance in the groups compared. Pairs of independent groups were compared using the MannWhitney U test. Three independent groups were compared using the Kruskal-Wallis ANOVA followed by multiple post hoc comparisons. Friedmann's ANOVA with post hoc tests (Wilcoxon's signed rank tests) were used to show differences among more than two dependent variables.

\section{RESULTS}

Three subgroups of depressed patients were identified on the basis of non-hierarchical cluster analysis. These subgroups differed in the dynamics of changes in KYNA concentrations measured before ECT and after 1, 6 and 12 ECT sessions. Cluster analysis leads to the grouping of objects based on their mathematically determined similarity. In this study k-means clustering was used. It is a method in which clusters are formed in such a way that the mean distance between all the cases in the cluster is as short as possible. In other words, the method aims at maximizing similarity among the objects which form a cluster, minimizing intragroup variance and maximizing distances among clusters, i.e., minimizing intra-cluster variance and maximizing intercluster variance (Statistica 1997). Based on cluster analysis, the whole group of patients was divided into three subgroups with characteristic patterns of KYNA concentrations measured before ECT treatment and after 1, 6 and 12 ECT sessions (Figure 1).

Statistically significant differences among the three subgroups of patients with depression were found for blood plasma KYNA concentrations determined both before treatment and after 1, 6 and 12 ECTs. Subgroup III patients had significantly lower concentrations of KYNA than subgroup I patients before ECT and after 1 and 6 ECT sessions. Patients from subgroup II had significantly higher concentrations of KYNA than patients from subgroups I and III before ECT and after 6 and 12 ECT sessions (Table 1, Table 2).

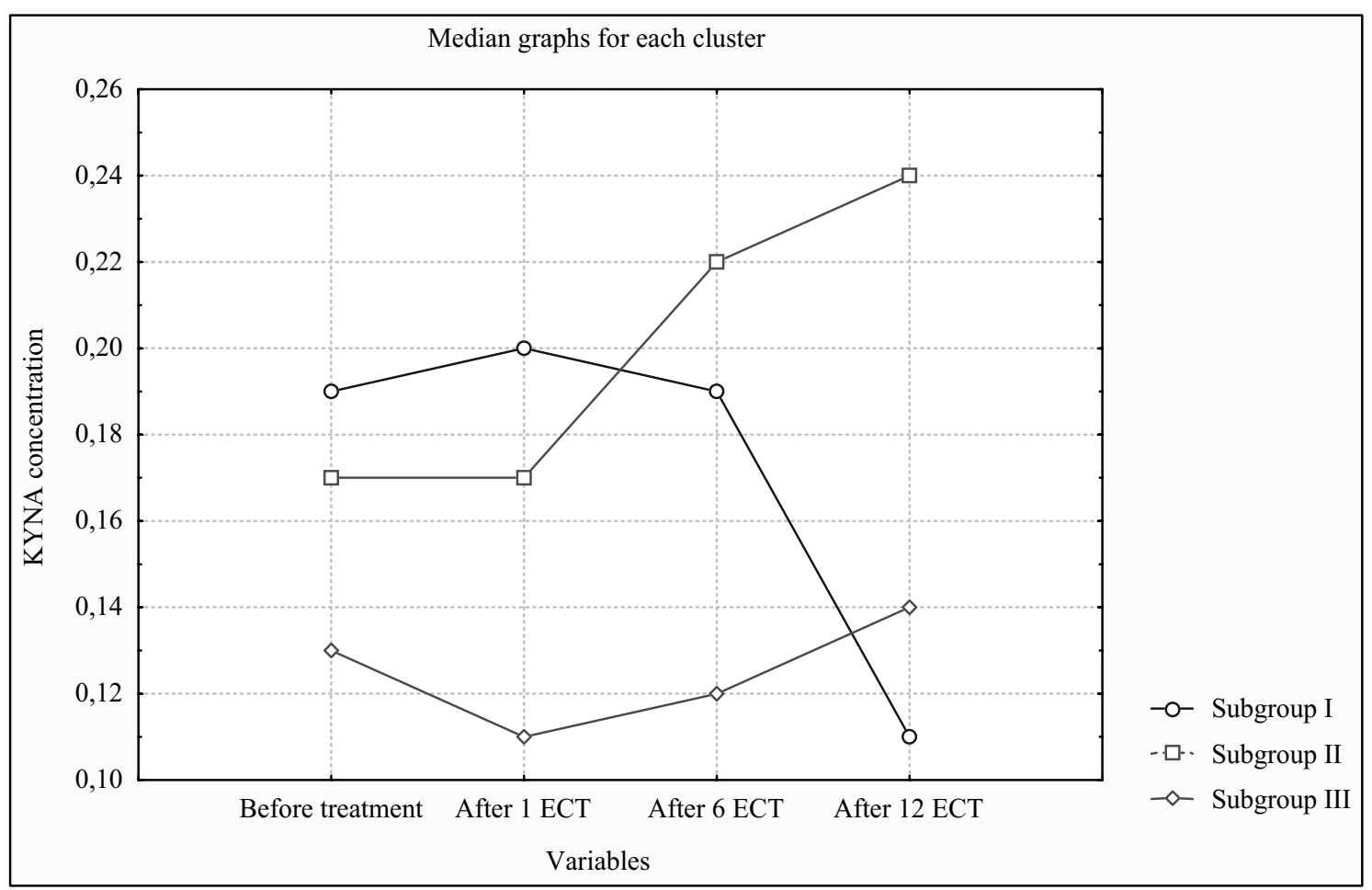

Figure 1. Median KYNA concentrations determined in patients from the three subgroups before treatment and after 1, 6 and 12 ECTs 
Table 1. Comparison of KYNA concentrations in three subgroups of patients with depression (Kruskal-Wallis ANOVA tests)

\begin{tabular}{|c|c|c|c|c|c|c|c|c|c|c|c|}
\hline \multirow{2}{*}{$\begin{array}{l}\text { KYNA } \\
\text { concentration }\end{array}$} & \multicolumn{3}{|c|}{ Subgroup I } & \multicolumn{3}{|c|}{ Subgroup II } & \multicolumn{3}{|c|}{ Subgroup III } & \multirow{2}{*}{$\mathrm{H}$} & \multirow{2}{*}{$\mathrm{p}$} \\
\hline & Median & Min. & Max. & Median & Min. & Max. & Median & Min. & Max. & & \\
\hline Before treatment & 0.19 & 0.13 & 0.45 & 0.17 & 0.06 & 0.22 & 0.13 & 0.02 & 0.19 & 9.39 & 0.010 \\
\hline After 1 ECT & 0.20 & 0.14 & 0.29 & 0.17 & 0.06 & 0.27 & 0.11 & 0.08 & 0.16 & 9.59 & 0.010 \\
\hline After 6 ECTs & 0.19 & 0.15 & 0.23 & 0.22 & 0.20 & 0.34 & 0.12 & 0.05 & 0.18 & 19.29 & 0.001 \\
\hline After 12 ECTs & 0.11 & 0.04 & 0.19 & 0.24 & 0.17 & 0.28 & 0.14 & 0.08 & 0.23 & 12.93 & 0.002 \\
\hline
\end{tabular}

Table 2. Levels of significance of differences among KYNA concentrations in the blood plasma of patients from the three subgroups studied (Kruskal-Wallis ANOVA tests)

\begin{tabular}{lccc}
\hline & & Subgroups & II-III \\
\hline KYNA concentration & I-II & I-III & not significant \\
Before treatment & not significant & 0.003 & not significant \\
After 1 ECT & not significant & 0.001 & 0.001 \\
After 6 ECTs & 0.02 & 0.002 & 0.003 \\
After 12 ECTs & 0.002 & not significant & \\
\hline
\end{tabular}

Table 3. Levels of significance of differences among KYNA concentrations in the three subgroups of patients ( results of Friedmann test)

\begin{tabular}{|c|c|c|c|c|c|c|}
\hline \multirow[b]{2}{*}{ Subgroups } & \multicolumn{6}{|c|}{ KYNA concentration } \\
\hline & $\begin{array}{l}\text { Before } \\
\text { treatment - } \\
\text { after } 1^{\text {st }} \mathrm{ECT}\end{array}$ & $\begin{array}{l}\text { Before } \\
\text { treatment }- \\
\text { after } 6^{\text {th }} \text { ECT }\end{array}$ & $\begin{array}{l}\text { Before treatment } \\
- \text { after } 12^{\text {th }} \mathrm{ECT}\end{array}$ & $\begin{array}{l}\text { After } 1^{\text {st }} \\
\text { ECT-after } 6^{\text {th }} \\
\text { ECT }\end{array}$ & $\begin{array}{l}\text { After } 1^{\text {st }} \\
\text { ECT-after } \\
12^{\text {th }} \text { ECT }\end{array}$ & $\begin{array}{l}\text { After } 6^{\text {th }} \text { ECT- } \\
\text { after } 12^{\text {th }} \text { ECT }\end{array}$ \\
\hline Subgro & not significant & not significant & 0.01 & not significant & 0.02 & 0.02 \\
\hline Subgroup II & not significant & 0.01 & 0.02 & 0.04 & 0.01 & not significant \\
\hline Subgroup III & not significant & not significant & not significant & not significant & not significant & not significant \\
\hline
\end{tabular}

Next, the dynamics of changes in KYNA concentrations before treatment and after 1, 6 and 12 ECTs was analyzed in patients from the three subgroups. Friedman's ANOVA and Kendall's coefficient of concordance were used to assess whether the changes in the concentrations of KYNA in patients from the three subgroups were statistically significant. Levels of significance of differences among KYNA concentrations determined before treatment and after 1, 6 and 12 ECTs were calculated using Wilcoxon's signed rank test (Table 3).

Changes in the concentration of KYNA in subgroup I were statistically significant $\left(\chi^{2}=10.20 ; \mathrm{df}=3 ; \mathrm{p}<0.02\right)$. Blood serum concentrations of KYNA in subgroup I patients, determined after 12 ECTs were significantly lower compared to its pre-therapy concentrations and to the concentrations of KYNA measured after 1 and 6 ECTs. Blood serum concentrations of KYNA in subgroup II patients, determined before treatment and after 1,6 and 12 ECTs, underwent statistically significant changes $\left(\chi^{2}=13.05 ; \mathrm{df}=3 ; \mathrm{p}<0.01\right)$. Although there were no changes in KYNA concentrations in the serum of patients from subgroup II measured before treatment and after one ECT - after 6 and 12 ECT sessions KYNA concentrations increased significantly compared to baseline and compared to the level determined after one ECT. Blood serum concentrations of KYNA in subgroup III patients, determined before treatment and after
1, 6 and 12 ECTs, underwent no statistically significant changes $\left(\chi^{2}=1.70 ; \mathrm{df}=3 ; \mathrm{p}<0.64\right)$. In this subgroup, changes in the concentration of KYNA were the least dynamic.

As a next step in the analysis, the Mann-Whitney U test was used to compare KYNA levels in the blood plasma of ECT-treated patients from the three subgroups with the concentrations of KYNA in the control group (Tables 4-6).

Blood serum KYNA concentrations in subgroup I patients before treatment and after 1 and 6 ECTs were not significantly different from those of control subjects, but after 12 ECT sessions, they were significantly lower than in healthy subjects.

KYNA concentrations in the blood plasma of subgroup II patients before ECT therapy and after 1, 6 and 12 ECT sessions were not significantly different from KYNA levels in the control group.

KYNA concentrations in the blood serum of patients from subgroup III, both before ECT treatment and after 1, 6 and 12 ECT sessions were significantly lower than the concentrations of KYNA in healthy subjects.

The three subgroups of patients were compared, using the Kruskal-Wallis test, for age, number of phases of the disease, duration of symptoms, duration of a phase, and scores on the clinical scales used - MADRS, CGI and the Beck Depression Inventory (Table 7). 
Table 4. Comparison of blood plasma KYNA concentrations in subgroup I patients and controls (differences vs control group)

\begin{tabular}{lccrc}
\hline KYNA concentration & Median & Z & $\mathrm{p}$ \\
\hline Before treatment & Control Group & Subgroup I & -0.04 & 0.972 \\
After 1 ECT & 0.20 & 0.19 & 0.05 & 0.963 \\
After 6 ECTs & 0.20 & 0.19 & 0.32 & 0.752 \\
After 12 ECTs & 0.20 & 0.11 & 2.74 & 0.006 \\
\hline
\end{tabular}

Table 5. Comparison of blood plasma KYNA concentrations in subgroup II patients and controls (differences vs control group)

\begin{tabular}{|c|c|c|c|c|}
\hline \multirow{2}{*}{ KYNA concentration } & \multicolumn{2}{|c|}{ Median } & \multirow{2}{*}{$\mathrm{Z}$} & \multirow[b]{2}{*}{$\mathrm{p}$} \\
\hline & Control Group & Subgroup II & & \\
\hline Before treatment & 0.20 & 0.17 & 1.05 & 0.292 \\
\hline After 1 ECT & 0.20 & 0.17 & 1.62 & 0.106 \\
\hline After 6 ECTs & 0.20 & 0.22 & -0.98 & 0.325 \\
\hline After 12 ECTs & 0.20 & 0.24 & -0.80 & 0.426 \\
\hline
\end{tabular}

Table 6. Comparison of blood plasma KYNA concentrations in subgroup III patients and controls (differences vs control group)

\begin{tabular}{lcccc}
\hline KYNA concentration & Median & Z & p \\
\hline Before treatment & 0.20 & Subgroup III & 0.13 & 2.84 \\
After 1 ECT & 0.20 & 0.11 & 3.20 & 0.005 \\
After 6 ECTs & 0.20 & 0.12 & 3.01 & 0.001 \\
After 12 ECTs & 0.20 & 0.14 & 2.10 & 0.003 \\
\hline
\end{tabular}

Table 7. Comparison of subgroups in terms of age, disease duration, number of phases of the disease, and duration of relapse

\begin{tabular}{lccccc}
\hline Variables & Subgroup I & $\begin{array}{c}\text { Median } \\
\text { Subgroup II }\end{array}$ & Subgroup III & H & $\mathrm{p}$ \\
\hline Age & 49 & 48 & 53.5 & 1.88 & not significant \\
Number of phases & 3.5 & 4 & 4 & 0.65 & not significant \\
Duration of symptoms (in years) & 5 & 12.5 & 11 & 2.93 & not significant \\
Duration of relapse (in years) & 6 & 5 & 4 & 0.35 & not significant \\
MADRS & 37.5 & 42.5 & 36 & 6.17 & not significant \\
CGI & 5 & 5 & 5 & 5.04 & not significant \\
BECK & 35 & 41.5 & 37.5 & 3.28 & not significant \\
\hline
\end{tabular}

Patients from the three subgroups did not differ in age, duration of the disease, number of phases of the disease or duration of relapse.

Summarizing the results of cluster analysis, it should be noted that subgroup I comprised 8 patients, with more men $(62.50 \%)$ than women $(37.50 \%)$. This subgroup had the highest levels of blood serum KYNA before ECT treatment and after one ECT session, and the lowest levels of KYNA after 12 ECTs compared to subgroups II and III. In this subgroup, $50 \%$ of patients showed clinical improvement as measured by MADRS, i.e. fewer than in subgroups II and III.

In subgroup II, there were 8 patients, with more women $(75 \%)$ than men $(25 \%)$. Clinical improvement was observed in $75 \%$ of the patients. Compared with the other two subgroups, the patients from subgroup II had the highest concentrations of KYNA after 6 and 12 ECTs.
Subgroup III consisted of 12 patients, with more women $(66.7 \%)$ than men $(33.33 \%)$. Compared to subgroups I and II, this subgroup had the largest number of patients who showed clinical improvement as measured on the MADRS scale (83.33\%). Blood plasma KYNA concentrations in subgroup III patients, measured before treatment and after 1 and 6 ECTs were lower in comparison to the other two subgroups. After 12 ECTs, KYNA concentrations in the blood serum of patients from subgroup III were slightly higher than those in the blood serum of patients from subgroup I. This was the only subgroup in which changes in KYNA concentrations prior to treatment, during ECT and after treatment were not statistically significant. It should be emphasized that the subgroups identified did not differ significantly in terms of age, gender, duration of symptoms, number of depressive phases, duration of a depressive phase, severity of depression or the general level of functioning. 
Patients with psychotic depression and non-psychotic depression were merged into one group. During the statistical analysis, it was found that the level of KYNA before tratment did not differ significantly between the groups of psychotic and non-psychotic depression. After treatment significant difference occurred only after 6th procedure of ECT. When the patients with psychotic depression were excluded from the analysis, the statistical significance of the results was not altered.

\section{DISCUSSION}

In this study, three subgroups of depressed patients were identified, which differed in blood plasma concentrations of KYNA before ECT treatment and in the dynamics of changes in KYNA concentrations during ECT and after treatment. The subjects did not differ significantly in terms of age, gender, duration of symptoms, number of depressive phases and duration of the depressive phase. The largest number of patients with clinical improvement $(83 \%)$ were found in group III, which was characterized by the lowest blood plasma concentrations of KYNA before ECT and after the first and sixth ECTs. These concentrations were significantly lower than those determined in healthy individuals. Subgroup III was characterized by the lowest dynamics of changes in KYNA concentrations compared to subgroups I and II, since it was only in this subgroup of patients that no statistically significant changes in the concentrations of KYNA, measured before, during and after ECT, were found. KYNA concentrations in the blood serum of patients from subgroup III, both before ECT and after 1, 6 and 12 ECT sessions were significantly lower than the concentrations of KYNA in healthy subjects.

Subgroup I included the smallest number of patients with clinical improvement $(50 \%)$, as measured by MADRS. In this subgroup, there were more men than women (in contrast to the other two subgroups), and KYNA levels in the blood plasma of the subjects were significantly higher than those in the blood serum of subgroup III patients, both before ECT and after 1 and 6 ECT sessions. The concentrations of KYNA in subgroup I patients determined after 12 ECTs were significantly lower compared to the baseline level and to the concentrations measured after 1 and 6 ECTs. Blood serum KYNA concentrations in subgroup I patients before treatment and after 1 and 6 ECTs were not significantly different from those of control subjects; and after 12 ECT sessions, they were significantly lower than in healthy subjects. Patients from subgroup I had the lowest blood serum concentrations of KYNA after treatment, as compared to subgroup III and, especially, subgroup II.

At baseline and after one ECT session, the concentrations of KYNA in patients from subgroup II were higher than those in subgroup III patients, but lower than those in subgroup I patients. After 6 ECT sessions and after completion of the treatment, however, subgroup II had significantly higher KYNA levels than either subgroup I or III. Clinical improvement was observed in $75 \%$ of patients from subgroup II. KYNA concentrations in the blood plasma of subgroup II patients before ECT therapy and after 1, 6 and 12 ECT sessions were not significantly different from KYNA levels in the control group.

According to Olajossy (2010), clinical improvement can be predicted in people (especially women) with lower baseline concentrations of KYNA.

The findings reported above are consistent with previous results obtained by Olajossy (2010), who emphasizes that patients with recurrent depressive disorder can be divided into subgroups in which changes in the level of KYNA may constitute a therapeutic clue and allow accurate prediction of changes in blood serum concentrations of KYNA after ECT treatment.

The results obtained in the present study allow us to formulate the hypothesis that people with significantly lower baseline levels of KYNA, compared to healthy subjects, are characterized by a stable concentration of KYNA during ECT treatment, and the largest number of patients in this group achieve clinical improvement after the final (twelfth) ECT session.

On the other hand, patients whose pre-treatment KYNA levels do not differ significantly from those of the control group, should be monitored for their clinical condition and for the concentration of KYNA after six ECT sessions, because in the case of an increase in KYNA levels in this period, relative to baseline values, one can predict clinical improvement in a larger number of patients; a smaller number of patients will show clinical improvement in the situation when the concentration of KYNA decreases after the sixth ECT. Such a procedure may enable earlier modification of therapy in patients with poor response to ECT after six ECT sessions.

We can find an interesting study, where authors found the increased KYNA, KYN/TRP ratio, KYNA/KYN ratio and $\mathrm{KYNA} / 3$-hydroxykynurenine ratio over time during the period of the study. Furthermore these authors observed that KYN and KYN/TRP were negatively associated with total score of HDRS over time. Time to ECT response was not predicted by baseline TRP metabolite concentrations (Guloksuz et al. 2015). Other authors presented an altered kynurenine metabolism in these patients displayed by decreased plasma levels of KYNA and by a significantly increased QUIN/KYNA ratio. Levels of tryptophan, kynurenine and QUIN was not different between patients and controls. There was an association between treatment with ECT and a significant decrease in the plasma levels of tryptophan, kynurenine, and QUIN, but plasma KYNA levels did not change. The QUIN/KYNA ratio was found to significantly decrease in ECT-treated patients $(\mathrm{P}<0.05)$. Furthermore authors found a significant inverse correlation between symptom severity and kynurenine levels at baseline (Schwieler et al. 2016). Undoubtedly, there is a need to extend the study group and deepen research. 


\section{CONCLUSIONS}

- The largest number of patients with clinical improvement $(83 \%)$ was observed in the subgroup with significantly lower pre-treatment, treatment and post-treatment KYNA concentrations than those determined in the control group and with the lowest dynamics of changes in KYNA concentrations during ECT.

- Clinical improvement was observed in $75 \%$ of depressed patients who had insignificantly lower pre-treatment levels of KYNA and slightly higher levels of this acid after 6 and 12 ECT sessions than controls.

- The smallest number of patients with clinical improvement $(50 \%)$ was observed in the subgroup of patients who had similar pre-treatment and treatment concentrations of KYNA to controls and whose post-treatment concentrations of KYNA were significantly lower than those of healthy individuals.

- Due to low number of patients the chi-square analysis does not show significant differences between the percentages of patients in each group showing good response to ECT.

\section{Acknowledgements:}

This research was funded as part of the statutory activity of the Medical University of Lublin, research topic No. 191.

\section{Conflict of interest: None to declare.}

\section{Contribution of individual authors:}

Marcin Olajossy: study design, first draft, approval of the final version;

Bartosz Olajossy: collection of research materials, approval of the final version;

Emilia Potembska: literature review, statistical analyses, interpretation of results;

Nikodem Skoczeń: literature review;

Sebastian Wnuk: collection of research materials, approval of the final version;

Ewa Urbańska: study design,collection of research materials.

\section{References}

1. Bobula B, Tokarski K, Hess G: Repeated administration of antidepressants decreases field potentials in rat frontal cortex. Neuroscience 2003;120:765-9

2. Campbell S, MacQueen G: An update on regional brain volume differences associated with mood disorders. Curr Opin Psychiatry 2006; 19:25-33

3. Chen B, Dowlatshahi D, MacQueen GM, Wang JF, Young LT: Increased hippocampal BDNF immunoreactivity in subjects treated with antidepressant medication. Biol Psychiatry 2001; 50:260-5
4. Diazgranados N, Ibrahim L, Brutsche NE, Newberg A, Kronstein $P$, Khalife $S$, et al.: A randomized add-on trial of an $\mathrm{N}$-methyl-D-aspartate antagonist in treatment-resistant bipolar depression. Arch Gen Psychiatry 2010; 67:793-802

5. Dong J, Min S, Wei K, Li P, Cao J, Li Y: Effects of electroconvulsive therapy and propofol on spatial memory and glutamatergic system in hippocampus of depressed rats. J ECT 2010; 26:126-130

6. Erhardt S, Schwieler L, Engberg G: Kynurenic acid and schizophrenia. Adv Exp Med Biol 2003; 527:155-165

7. Guloksuz S, Arts B, Walter S, Drukker M, Rodriguez L, Myint AM, Schwarz MJ, Ponds R, van Os J, Kenis G, Rutten BPF: The impact of electroconvulsive therapy on the tryptophan-kynurenine metabolic pathway Brain Behav Immun 2015; 48:48-52

8. Guy W: ECDEU Assessment manual for psychopharmacology, revised, US Department of Health, Education, and Welfare, ADDAMHA, NIMH, Rockville 1976; 217-22

9. Fumagalli $F$, Pasini $M$, Sartorius A, et al.: Repeated electroconvulsive shock (ECS) alters the phosphorylation of glutamate receptor subunits in the rat hippocampus. Int J Neuropsychopharmacol 2010; 13:1255-1260

10. Hilmas C, Pereira EF, Alkondon M, Rassoulpour A, Schwarcz R, Albuquerque EX: The brain metabolite kynurenic acid inhibits alpha7 nicotinic receptor activity and increases non-alpha7 nicotinic receptor expression: physiopathological implications. J Neurosci 2001; 21:7463-73

11. Kavalali ET \& Monteggia LM: Synaptic mechanisms underlying rapid antidepressant action of ketamine. Am J Psychiatry 2012; 169:1150-6

12. Konarski JZ, McIntyre RS, Kennedy SH, Rafi-Tari S, Soczynska JK, Ketter TA: Volumetric neuroimaging investigations in mood disorders: bipolar disorder versus major depressive disorder. Bipolar Disord 2008; 10:1-37

13. Koolschijn PC, van Haren NE, Lensvelt-Mulders GJ, Hulshoff Pol HE, Kahn RS: Brain volume abnormalities in major depressive disorder: a meta-analysis of magnetic resonance imaging studies. Hum Brain Mapp 2009; 30:3719-3735

14. Lorenzetti V, Allen NB, Fornito A, Yücel M: Structural brain abnormalities in major depressive disorder: a selective review of recent MRI studies. J Affect Disord 2009; 117:1-17

15. Maes M, Gatecki P, Verkerk R, Rief W: Somatization, but not depression, is characterized by disorders in the tryptophan catabolite (TRYCAT) pathway, indicating increased indoleamine 2,3-dioxygenase and lowered kynurenine aminotransferase activity. Neuro Endocrinol Lett 2011; 32:264-73

16. Michael N, Erfurth A, Ohrmann P, Arolt V, Heindel W, Pfleiderer B: Metabolic changes within the left dorsolateral prefrontal cortex occurring with electroconvulsive therapy in patients with treatment resistant unipolar depression. Psychol Med 2003; 33:1277-84

17. Montgomery SA \& Asberg M: A new depression scale designed to be sensitive to change. Br J Psychiatry 1979; 134:382-9

18. Myint AM: Kynurenines: from the perspective of major psychiatric disorders. FEBS J 2012; 279:1375-85

19. Myint AM \& Kim YK: Cytokine-serotonin interaction through IDO: a neurodegeneration hypothesis of depression. Med. Hypotheses 2003; 61:519-525 
20. Myint AM, Kim YK, Verkerk R, Scharpe S, Steinbusch H, Leonard B: Kynurenine pathway in major depression: evidence of impaired neuroprotection. J Affect Disord 2007; 98:143-51

21. Olajossy M: Poziom kwasu kynureninowego w surowicy chorych na depresje leczonych elektrycznie. Rozprawa habilitacyjna. Lublin: Uniwersytet Medyczny w Lublinie, 2010.

22. Olajossy M, Olajossy B, Potembska E, Skoczeń $N$, Wnuk S, Urbańska E: Stężenie kwasu kynureninowego $i$ wybranych cytokin podczas leczenia przeciwdepresyjnego. Neuropsych Neuropsychol 2014; 9:1-7

23. Orser BA, Pennefather PS \& MacDonald JF: Multiple mechanisms of ketamine blockade of N-methyl-D-aspartate receptors. Anesthesiology 1997; 86:903-917

24. Papp $M \&$ Moryl E: Antidepressant activity of noncompetitive and competitive NMDA receptor antagonists in a chronic mild stress model of depression. Eur $J$ Pharmacol 1994; 263:1-7

25. Pessoa L: On the relationship between emotion and cognition. Nat Rev Neurosci 2008; 9:148-158

26. Pittaluga A, Raiteri L, Longordo F, Luccini E, Barbiero VS, Racagni G, et al.: Antidepressant treatments and function of glutamate ionotropic receptors mediating amine release in hippocampus. Neuropharmacology 2007; 53:27-36

27. Price RB, Nock MK, Charney DS, Mathew SJ: Effects of intravenous ketamine on explicit and implicit measures of suicidality in treatment-resistant depression. Biol Psychiatry 2009; 66:522-6

28. Raol YH, Lynch DR, Brooks-Kayal AR: Role of excitatory amino acids in developmental epilepsies. Ment Retard Dev Disabil Res Rev 2001; 7:254-60

29. Rybakowski JK, Permoda-Osip A, Skibinska M, Adamski $R$, Bartkowska-Sniatkowska A: Single ketamine infusion in bipolar depression resistant to antidepressants: are neurotrophins involved? Hum Psychopharmacol 2013; 28:87-90

30. Schwieler L, Samuelsson M, Frye MA, Bhat M, SchuppeKoistinen I, Jungholm O, Johansson AG, Landén M, Sellgren CM, Erhardt S: Electroconvulsive therapy suppresses the neurotoxic branch of the kynurenine pathway in treatment-resistant depressed patients. Journal of Neuroinflammation 2016; 13:51. DOI: 10.1186/s12974-016-0517-7

31. Skolnick P, Layer RT, Popik P, Nowak G, Paul IA, Trullas $R$ : Adaptation of $N$-methyl-D-aspartate (NMDA) receptors following antidepressant treatment: implications for the pharmacotherapy of depression. Pharmacopsychiatry 1996; 29:23-6

32. Statistica, Kraków: StatSoft Polska; 1997, s. 3175

33. Thackray SJ, Mowat CG, Chapman SK: Exploring the mechanism of tryptophan 2,3-dioxygenase. Biochem Soc Trans 2008; 36:1120-3

34. Turski WA, Nakamura M, Todd WP, Carpenter BK, Whetsell WO Jr, Schwarcz $R$ : Identification and quantification of kynurenic acid in human brain tissue. Brain Res 1988; 454:164-9

35. Urbańska EM, Kocki T, Saran T, Kleinrok Z, Turski WA: Impairment of brain kynurenic acid production by glutamate metabotropic receptor agonists. Neuroreport 1997; 8:3501-5

36. Yamakura T, Askalany AR, Petrenko AB, Kohno T, Baba $H$ \& Sakimura K: The NR3B subunit does not alter the anesthetic sensitivities of recombinant $N$-methyl-D-aspartate receptors. Anesth Analg 2005; 100:1687-1692

37. Zarate CA Jr, Singh JB, Carlson PJ, Brutsche NE, Ameli $R$, Luckenbaugh DA, et al.: A randomized trial of an $N$ methyl-D-aspartate antagonist in treatment-resistant major depression. Arch Gen Psychiatry 2006; 63:856-64

38. Zyss T. elektrowstrzasy: wprowadzenie do bioelektrycznej natury zaburzeń depresyjnych. Warszawa: Elmico, 2009

Correspondence:

Emilia Potembska, MD, PhD

Department of Psychiatric Nursing Medical University of Lublin

ul. Gtuska 1, 20 - 439 Lublin, Poland

E-mail:ermila100@gmail.com 